\title{
Retropharyngeal lipoma with parapharyngeal extension: is transoral excision possible?
}

Dennis Yu Kim $\underline{\text { Chua }}^{1}$, mBBs, Mrcse, Ming Yann $\underline{\operatorname{Lim}}^{1}$, MBBS, MrCs, Dawn Tju Wei $\underline{\operatorname{TeO}}^{2}$, MBBS, FAMs, Siew Yoong $\underline{\mathrm{Hwang}^{3}}$, MBBS, FRCSG

\begin{abstract}
Retropharyngeal lipomas are rare tumours that are usually asymptomatic until they reach a large size. The definitive treatment is surgical excision. Since the tumours are typically large at the time of presentation, extensive surgery for complete clearance of the lipoma from the retropharyngeal and parapharyngeal regions is to be expected. Transoral excision is typically indicated for small retropharyngeal tumours, as this approach does not give good access to the parapharyngeal area laterally. Herein, we present the case of a patient who underwent transoral excision of a huge retropharyngeal lipoma, which extended into the right parapharyngeal space. The surgical technique used and the insights gained are described in this report. Even with parapharyngeal extension, transoral resection of a huge retropharyngeal lipoma can be performed. More invasive surgery, which may involve a neck incision, mandibulotomy or pharyngotomy, is not necessary. While huge retropharyngeal lipomas are usually symptomatic and require surgical intervention, transoral resection can be adequate and safe for treatment.
\end{abstract}

Keywords: lipoma, retropharyngeal, transoral excision

\section{INTRODUCTION}

Retropharyngeal lipomas are rare entities that are usually asymptomatic until they reach a large size. Clinical presentation varies, depending on the portion of the aerodigestive tract affected.(1) Investigations usually consist of either computed tomography $(\mathrm{CT})$ or magnetic resonance $(\mathrm{MR})$ imaging. Both $\mathrm{CT}$ and MR imaging can help with the diagnosis, as well as assessment of the extent of the lipoma. While fine-needle aspiration cytology (FNAC) can allude to the diagnosis, definitive diagnosis depends on histological confirmation, which can only be obtained via surgical excision. Various approaches for surgical excision have been described in the literature, with the transcervical approach being the most common for larger masses. ${ }^{(2)}$ In this report, we describe the use of the transoral approach to achieve complete resection of a huge retropharyngeal lipoma with parapharyngeal extension. Surgical excision via the transoral approach was performed safely and adequately.

\section{CASE REPORT}

A 71-year-old Chinese man with a medical history of hyperlipidaemia presented to our institution with a right cerebellar bleed that was conservatively managed by a neurologist. CT revealed an incidental finding of a homogeneous mass measuring $9.4 \mathrm{~cm} \times 6.7 \mathrm{~cm}$ in the posterior pharyngeal wall, extending from the postnasal space to the hypopharynx, which was suggestive of a lipoma (Fig. 1). The lipoma extended from the $\mathrm{C} 1$ to $\mathrm{C} 6$ vertebral body. MR image showed a lipoma arising from the right prevertebral space, extending to the parapharyngeal space and displacing the carotid sheath (Fig. 2).

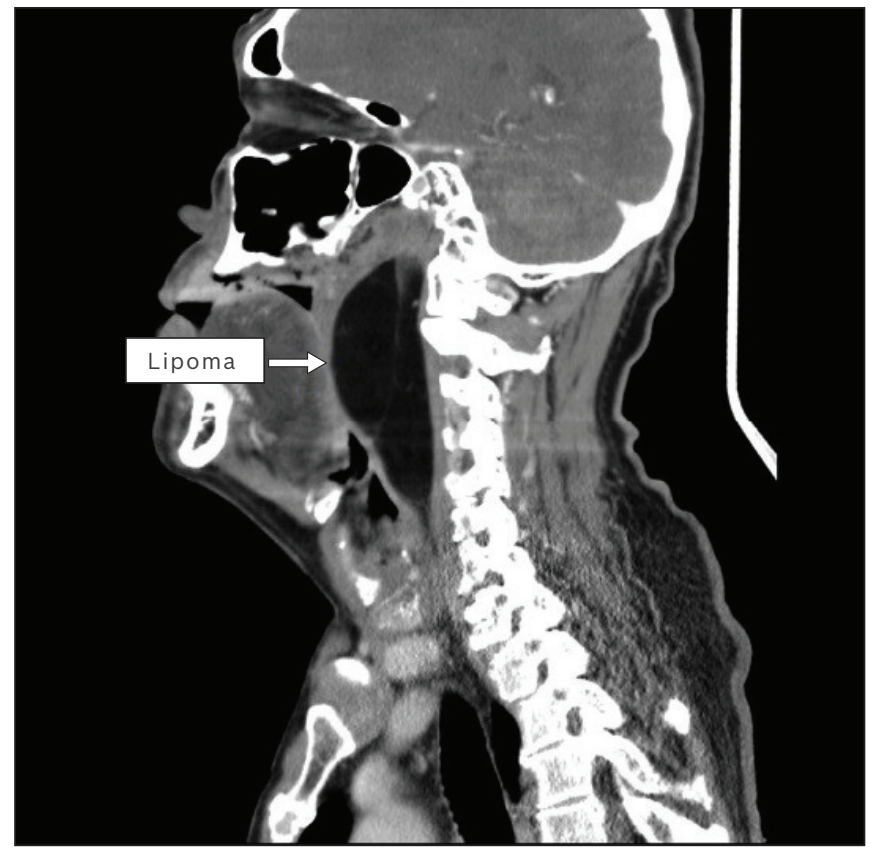

Fig. 1 CT image of the head and neck shows a homogeneous mass measuring $9.4 \mathrm{~cm} \times 6.7 \mathrm{~cm}$ in the posterior pharyngeal wall, extending from the postnasal space to the hypopharynx (from C1 to C6).

The patient's only complaint was mild dysphagia. Treatment options were presented to the patient and he elected for surgical excision of the tumour, for fear that it would increase in size and worsen his dysphagia, possibly resulting in the need for a more morbid operation in the future.

The patient underwent transoral surgical excision of the huge retropharyngeal lipoma. The operation was performed with the patient under general anaesthesia. After a Boyle-Davis gag

${ }^{1}$ Department of Otorhinolaryngology, Tan Tock Seng Hospital, ${ }^{2}$ Department of Otolaryngology, KK Women's and Children's Hospital, ${ }^{3}$ Care Sinus Snoring ENT Centre, Gleneagles Medical Centre, Singapore

Correspondence: Dr Chua Yu Kim Dennis, Associate Consultant, Department of Otorhinolaryngology, Tan Tock Seng Hospital, 11 Jalan Tan Tock Seng, Singapore 308433. dennis.chua.yk@gmail.com 


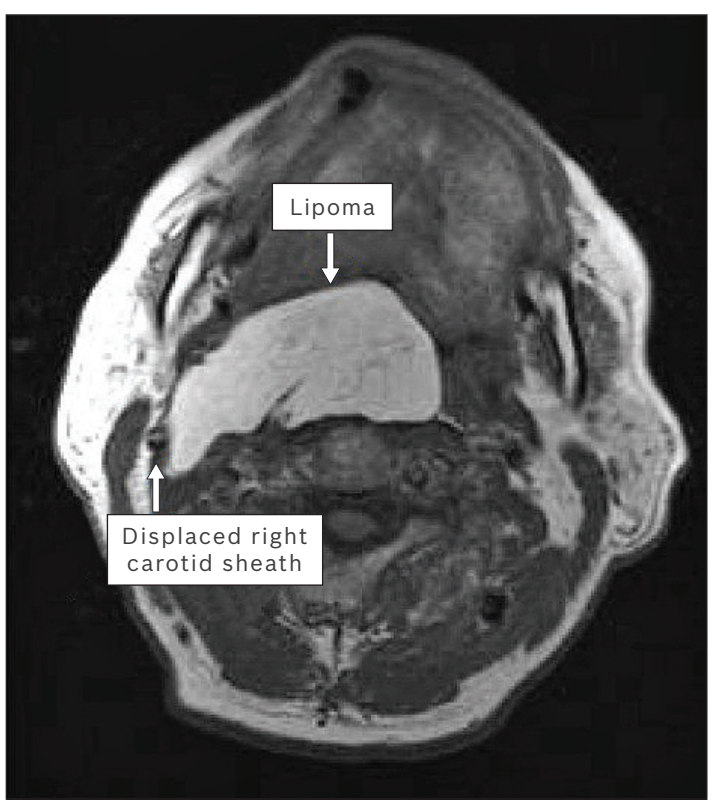

Fig. $2 M R$ image of the neck shows a lipoma arising from the right prevertebral space, extending to the parapharyngeal space and displacing the carotid sheath.

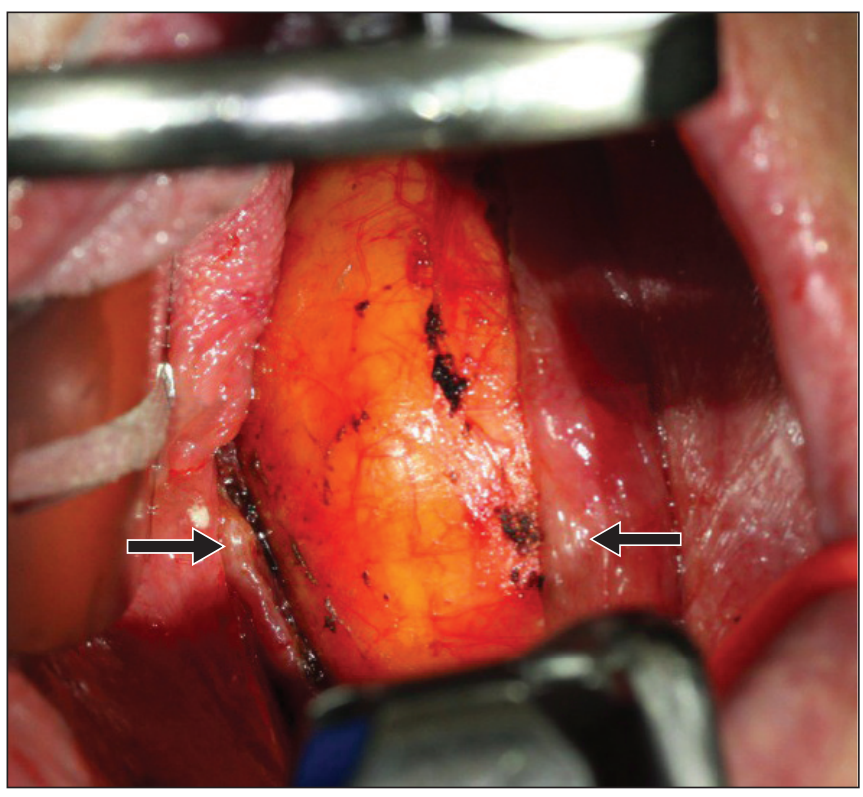

Fig. 3 Intraoperative photo shows the midline incision made over the lipoma via a transoral approach, and mucosal flaps (arrows) elevated laterally on both sides of the lipoma with the Boyle-Davis gag in situ.

was inserted, a midline incision was made over the lipoma and the mucosal flaps were elevated laterally on both sides (Fig. 3). Once the mucosal flaps were elevated, the lipoma was seen bulging toward the oropharynx. The lipoma was then freed from the surrounding mucosa and underlying prevertebral fascia using a combination of diathermy and blunt finger dissection. At the most lateral extent of the parapharyngeal space where visualisation was poor, blunt finger dissection was performed judiciously, keeping in mind the close proximity of the carotid vessels. The lipoma was then freed from the surrounding tissues and removed en bloc through the patient's mouth. As the lipoma was well-encapsulated and distensible, blunt dissection was easy to perform and en bloc transoral

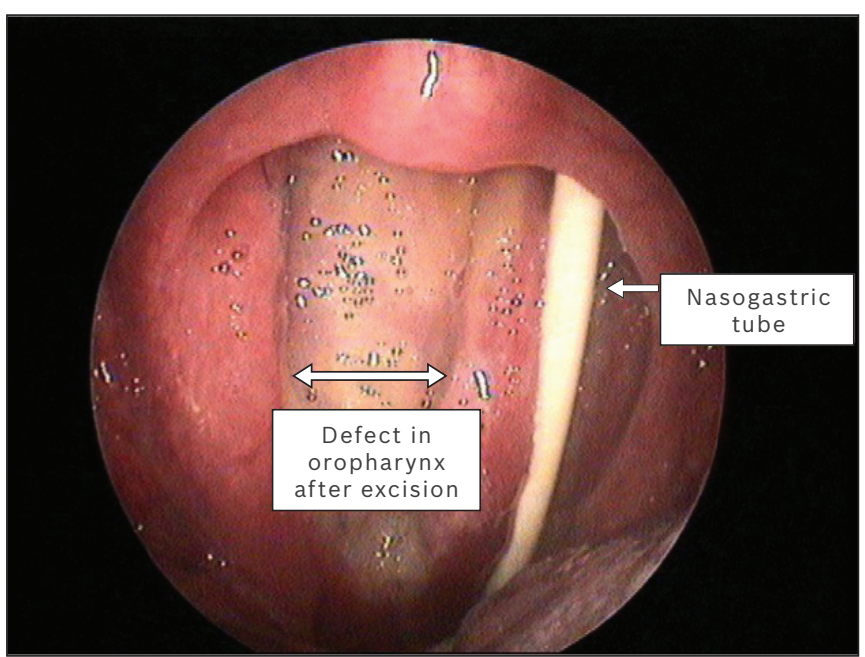

Fig. 4 Endoscopic view of the oropharynx taken with a $4-m m, 0^{\circ}$ endoscope shows the resultant defect after excision. This defect was left to heal via secondary intention and the patient was fed via a nasogastric tube for a week.

removal was possible. The defect was left open to heal by secondary intention (Fig. 4), and the patient was fed via a nasogastric tube for a week.

\section{DISCUSSION}

Retropharyngeal lipomas are rare tumours, with only about 30 cases reported in the literature since 1877.(1) Lipomas are slow-growing tumours that are usually not detectable until they reach a large size. As lipomas can extend from the base of the skull to the superior mediastinum, the symptoms can vary depending on the affected part of the aerodigestive tract. Common nonspecific clinical presentations that have been described include globus pharyngis, dysphagia and obstructive sleep apnoea with snoring or stertor. ${ }^{(1-3)}$ The exact location of the lipoma determines subsequent management.

The radiological features of a lipoma can be ascertained using either CT or MR imaging. Lipomas appear as homogeneous low-density areas of -60 to -120 Hounsfield units on nonconstrast-enhanced CT. On MR imaging, lipomas appear as a high-intensity signal on T1-weighted and T2weighted fast spin-echo sequences. ${ }^{(4)}$ Additional information such as the relationship between the lipoma and the prevertebral muscles, as well as the possibility of a liposarcoma, can also be obtained using MR imaging. ${ }^{(5)}$ Thus, we suggest that $M R$ imaging be used as the investigation of choice for retropharyngeal lipomas, if resources are available.

Since FNAC may not differentiate between a welldifferentiated liposarcoma and a lipoma, ${ }^{(6)}$ definite histology should be obtained via complete excision. This is especially the case for large retropharyngeal lipomas, as liposarcomas typically originate from the deep soft tissue. As large retropharyngeal lipomas usually result in the compression of the aerodigestive tract, leading to prominent symptoms, surgical resection is the mainstay of treatment. Furthermore, even if a retropharyngeal lipoma is asymptomatic, it will inevitably 
result in distressing symptoms due to its location. Therefore, we suggest that surgical excision be performed on all young patients with retropharyngeal lipomas, even if they are asymptomatic. The exception would be patients who are not fit for surgery and elderly patients with small lipomas.

A transcervical approach is usually employed for huge retropharyngeal lipomas. ${ }^{(2)}$ This approach gives wider access, especially to the lateral aspect of the parapharyngeal space, thus facilitating complete removal of the tumour. However, there is significant morbidity associated with the transcervical approach, including the risk of damaging cranial nerves and carotid vessels. In the present study, we have shown that it is possible to completely remove a huge retropharyngeal lipoma with parapharyngeal extension via a transoral approach. This is possible because lipomas are usually well-encapsulated and distensible.

In conclusion, a huge retropharyngeal lipoma is a rare entity with varied clinical presentations. Therefore, a high index of suspicion is necessary in order to make the diagnosis. Imaging modalities and FNAC are useful adjuncts in the workup of patients with huge retropharyngeal lipomas, and surgical excision is the definitive management. We propose transoral excision as the preferred approach for surgical removal of retropharyngeal lipomas, even large ones. This is due to the relative ease of the procedure and its lower postoperative morbidity compared to more invasive open approaches.

\section{REFERENCES}

1. Yoshihara T, Kawano K, Mita N. Retropharyngeal lipoma causing severe dysphagia and dyspnea. J Otolaryngol 1998; 27:363-6.

2. Namyslowski G, Scierski W, Misiolek M, Urbaniec N, Lange D. Huge retropharyngeal lipoma causing obstructive sleep apnea: A case report. Eur Arch Otorhinolaryngol 2006; 263:738-40.

3. Akhtar J, Shaykhon M, Crocker J, D'Souza AR. Retropharyngeal lipoma causing dysphagia. Eur Arch Otorhinolaryngol 2001; 258:458-9.

4. Dooms GC, Hricak H, Sollitto RA, Higgins CB. Liposarcomatous tumours and tumours with fatty components: MR imaging potential and comparisons between MR and CT results. Radiology 1985; 157:479-83.

5. Senchenkov A, Werning JW, Staren ED. Radiographic assessment of the infiltrating retropharyngeal lipoma. Otolaryngol Head Neck Surg 2001; 125:658-60.

6. Nemanqani D, Mourad WA. Cytomorphologic features of fine needle aspiration of liposarcoma. Diagn Cytopathol 1999; 20:67-9. 